\title{
Belphégor
}

\section{Les limites d'un roman : pratiques transfictionnelles dans l'œuvre de Fortuné du Boisgobey}

Nicolas Gauthier

\section{(2) OpenEdition}

Journals

Édition électronique

URL : https://journals.openedition.org/belphegor/3743

DOI : 10.4000/belphegor.3743

ISSN : 1499-7185

Éditeur

LPCM

Référence électronique

Nicolas Gauthier, «Les limites d'un roman : pratiques transfictionnelles dans l'œuvre de Fortuné du Boisgobey », Belphégor [En ligne], 19-1 | 2021, mis en ligne le 26 juin 2021, consulté le 06 juillet 2021. URL : http://journals.openedition.org/belphegor/3743 ; DOI : https://doi.org/10.4000/belphegor.3743

Ce document a été généré automatiquement le 6 juillet 2021.

\section{(c)}

Belphégor est mis à disposition selon les termes de la Licence Creative Commons Attribution - Pas d'Utilisation Commerciale - Pas de Modification 4.0 International. 


\title{
Les limites d'un roman : pratiques transfictionnelles dans l'œuvre de Fortuné du Boisgobey
}

\author{
Nicolas Gauthier
}

1 Dans Ma vie aventureuse, Sir Arthur Conan Doyle expose ainsi la réflexion qui a mené à l'élaboration de Sherlock Holmes :

Considérant ces divers recueils d'histoires détachées, je songeais qu'un même personnage circulant à travers une série de nouvelles, s'il parvenait à capter l'attention du lecteur, l'enchaînerait à son magazine ${ }^{1}$.

Quelques décennies auparavant, en France, Émile Gaboriau - évoqué par Holmes et Watson dans Une étude en rouge - avait aussi proposé avec son Monsieur Lecoq un détective exceptionnel récurrent. Bien qu'il s'agisse de romans-feuilletons - et non de nouvelles dans un magazine, comme la plupart des aventures de Holmes - et que l'importance de Lecoq varie considérablement à travers les œuvres ${ }^{2}$, Gaboriau avait ainsi opté pour une stratégie fonctionnant de façon analogue. Si son personnage ne connut pas l'immense popularité de Holmes, il eut néanmoins un vaste retentissement jusque dans le $\mathrm{xx}^{\mathrm{e}}$ siècle.

Ces deux figures célèbres illustrent que les constructions transfictionnelles s'imposent comme un trait générique au cœur du développement du roman judiciaire et de l'émergence, à la fin du $\mathrm{xIX}^{\mathrm{e}}$ siècle, du récit policier ${ }^{3}$. Bien que, durant les décennies 1870 et 1880, on ne trouve en France aucun cas comparable à Lecoq et Holmes - par la notoriété ou le nombre de titres impliquant un même personnage -, certaines œuvres proposent un univers fictionnel commun, selon une logique tenant généralement davantage du cycle que de la série 4 . Parmi les écrivains qui ont exploré cette avenue, Fortuné du Boisgobey (1821-1891) se distingue tout particulièrement par sa manière de retravailler ce trait générique selon une approche qui le distingue nettement de Gaboriau et de Conan Doyle. En effet, dans plusieurs récits publiés entre 1875 et 1885 , Boisgobey s'engage résolument dans une autre voie, puisque les procédés transfictionnels auxquels il a recours ne mettent pas en place un univers fictionnel 
cohérent qui engloberait plusieurs romans grâce à la présence d'un tel enquêteur récurrent; ils invitent plutôt le lecteur à s'interroger sur le statut de ce qu'il lit. Un questionnement inscrit dans une approche poétique s'impose donc ici pour comprendre comment ce romancier en vient à réactualiser ces codes qui forment une des principales fondations du genre, une réactualisation qui donne à voir autant sa maitrise desdits codes que ses efforts pour les remanier d'une manière qui lui est propre.

\section{Face à Gaboriau}

4 Le dernier quart du xIX siècle est friand d'«expansions transfictionnelles " ${ }^{5}$. Paul Mahalin, nom de plume d'Émile Blondet, publie Le Fils de Porthos en 1883, D'Artagnan en 1890, Le Filleul d'Aramis en $1896^{6}$ et Mademoiselle Monte-Cristo, aussi en 1896. Jules Lermina puise également dans l'œuvre dumasienne avec Le Fils de Monte-Cristo (1881) et Le Trésor de Monte-Cristo $(1885)^{7}$ tandis que Constant Guéroult utilise « des notes [de Ponson du Terrail] pour écrire des suites aux aventures de Rocambole (Le Retour de Rocambole et Les Nouveaux Exploits de Rocambole publiés en 1875-1877 dans La Petite Presse $^{8}$ ». Et Fortuné du Boisgobey reprend en 1877 l'enquêteur Lecoq de Gaboriau.

5 Romancier aujourd'hui méconnu, Boisgobey n'est pas un jeune auteur naïf lorsqu'il en vient à vivre de sa plume : âgé de 48 ans, il a été fonctionnaire, a beaucoup exploré la vie nocturne parisienne et a dévoré trois héritages au jeu'. Selon un modèle balzacien bien connu, il a «perdu ses illusions» et embrasse la profession de feuilletoniste en assumant sa motivation pécuniaire. Il débute à l'automne 1868 dans Le Petit Journal, avec Les deux comédiens (30 août au 27 octobre), alors qu'y est publié le Monsieur Lecoq de Gaboriau (27 mai au 3 décembre). Dans ses romans historiques, mondains, sociaux et judiciaires, il use des règles du roman-feuilleton mais avec un «cynisme froidement dosé » et les "adapt[e] à sa propre morphologie d'aptitudes et d'intérêts ${ }^{10}$ ». Il faut aussi noter son inventivité pour mettre en scène des crimes dont l'explication n'apparaît qu'à la fin du roman, témoignant alors de l'ingéniosité du criminel, mais aussi, bien évidemment, de celle de l'auteur. Pensons à l'assassinat passé inaperçu bien que commis au su et au vu de tous dans un espace clos et bondé dans Le Crime de l'Omnibus, ou au meurtrier qui réussit à manipuler la perception du temps des témoins pour se donner un alibi dans Un coup de pouce. Auteur d'une soixantaine de romans en un peu plus de vingt ans (1868-1891), Boisgobey a connu un succès indéniable et a été traduit en Angleterre, aux États-Unis et au Japon.

6 C'est donc un feuilletoniste accompli, ayant publié depuis une dizaine d'années dans Le Petit Journal, Le Petit Moniteur universel du Soir, La Petite Presse et Le Figaro, qui redonne vie au personnage de Gaboriau avec La Vieillesse de M. Lecoq (Le Nouveau Journal, du 28 août 1877 au 6 janvier 1878; Dentu, 1878'11). L'intrigue s'ouvre avec l'arrestation d'un portefaix transportant une malle contenant un cadavre, présageant le célèbre cas de la malle sanglante de Millery (ou malle à Gouffé) qui animera l'actualité judiciaire en 1889-1890 ${ }^{12}$. Dans le roman, le commissionnaire, témoin et suspect, est sourd, muet, analphabète et incapable de comprendre le langage des signes. Après s'être épuisée en conjectures, la police sollicite M. Lecoq qui se borne à quelques conseils. Néanmoins, lorsque son propre fils est emprisonné pour ce meurtre, il se met en chasse pour débusquer le véritable coupable et se mesure aux forces de l'ordre, qui sont, elles, convaincues d'avoir écroué le responsable, et à Tolbiac Tinchebray, un détective venu 
d'Angleterre. Cette confrontation entre les nations évoque, de façon moins humoristique, celle que proposera quelques années plus tard Maurice Leblanc entre Arsène Lupin et Herlock Sholmès ${ }^{13}$.

7 Marchant dans les traces de Gaboriau ${ }^{14}$, Boisgobey reprend moins le jeune agent moqueur de L'Affaire Lerouge $\left(1866^{15}\right)$, que l'enquêteur " aux allures de chef de bureau " du Dossier $113\left(1867^{16}\right)$ et l'homme n'ayant « rien de remarquable ${ }^{17}$ » que son habileté à démêler les écheveaux les plus complexes de Monsieur Lecoq (1868). La Vieillesse de M. Lecoq propose donc une autre affaire à cet enquêteur, en le dotant d'un fils qui justifie son implication dans celle-ci. Le lecteur familier de l'œuvre de Gaboriau rencontre divers clins d'œil, comme la mention d'une péripétie de Monsieur Lecoq ${ }^{18}$ ou le surnom "père Tire-au-clair ${ }^{19}$ ". Boisgobey intègre ainsi son récit à l'univers fictionnel formé des cinq romans de Gaboriau où évolue Lecoq et où resurgissent divers acolytes et victimes ${ }^{20}$.

D'autres écrivains s'approprient l'enquêteur Lecoq dans les années subséquentes ${ }^{21}$. En 1885, William Busnach et Henri Chabrillat font paraître La Fille de M. Lecoq dans le Petit Parisien (du 16 juillet au 13 novembre) et Émile Blavet publie vingt ans plus tard Le Fils de M. Lecoq dans Le Gaulois (du 29 décembre 1907 au 12 avril 1908), où ce fils a recours aux talents d'un «clone du détective de Doyle ${ }^{22}$ ». Pour ajouter à la confusion, Le Cri du peuple a republié La Vieillesse de M. Lecoq du 25 mai au 3 octobre 1884, sous le titre Le Fils de M. Lecoq ${ }^{23}$. Aucune de ces œuvres ne tient compte des « autres » héritiers; chacun est présenté comme un enfant unique. Lecoq apparaît dans deux autres romans de 1886 : Le Secret de Pel, signé "M. Lecoq", pseudonyme employé par Adolphe Grippa, et, aux États-Unis, File 114 (A Sequel to File 113 by Emile Gaboriau) d'Ernest A. Young. Thierry Chevrier a aussi repéré Lecoq dans un fascicule de 1908, de même que sous la plume d'Abel Valabrègue en 1946 et de Jean Kéry en 1952.

9 L'enquêteur respecté de La Vieillesse de M. Lecoq se montre partiellement à la hauteur de sa réputation: il ne se laisse pas tromper par les preuves accumulées contre son fils, remarque des indices ayant échappé aux policiers et en induit des hypothèses complexes. Cela dit, Boisgobey met à l'honneur ses erreurs et ses errances. Si les tâtonnements de l'enquête sont au cœur du roman judiciaire et policier - on le sait, sauf exception, découvrir rapidement le coupable est narrativement contre-productif dans un récit à énigme -, Lecoq multiplie les scénarios qui forment des échafaudages intellectuels qui sont impressionnants à la lumière des détails sur lesquels ils sont fondés, mais qui s'écroulent à l'épreuve des faits. Plus que des fausses pistes inhérentes au genre, il y a là une critique du procédé consistant à identifier le meurtrier seulement grâce à un exercice d'agilité mentale. Si le Lecoq de Boisgobey finit par y arriver, ce n'est qu'en arpentant la ville et après plusieurs tentatives dont l'échec est mis de l'avant; il résout l'énigme par essais et erreurs.

10 Ce portrait ambivalent de Lecoq a suscité des réactions contrastées. Régis Messac le décrit comme "un vieillard pleurnicheur et facile à tromper ${ }^{24}$ » mais Roger Bonniot s'inscrit en faux contre cette lecture, rappelant que plusieurs personnages font l'éloge de ses talents et que certains "stratagèmes [fort efficaces] lui sont restés familiers ${ }^{25}$ ». Cette divergence illustre comment Boisgobey a su s'approprier ce personnage. De plus, ce geste, que l'on ne peut réduire à une paresse auctoriale ou à une stratégie éditoriale, est construit de façon à brouiller certaines frontières.

11 En effet, en reprenant Lecoq, Boisgobey offre une "expansion transfictionnelle ${ }^{26}$ », ajoutant à ce que l'on sait du personnage, mais aussi une «capture transfictionnelle». 
C'est-à-dire que le narrateur indique que son « M. Lecoq de Gentilly n'était autre que le fameux M. Lecoq, celui dont les exploits racontés par un éminent et regretté romancier ont charmé tant de lecteurs ${ }^{27}$ ». «Romancier », non biographe ou journaliste : il n'y a pas ici «occultation transfictionnelle», le lecteur est confronté à l'existence du texte antérieur qui a été "capturé" par celui-ci, dans un geste qui «sembl[e] à la fois proclamer la fictionnalité du roman original et la réalité de l'histoire qu'il relate ${ }^{28} »$. Est créée une relation non de fiction à fiction mais « entre une fiction et un texte : une fiction qui se donne pour plus englobante que celle dont elle dérive ${ }^{29}$ ». L'écrivain invite ainsi le lecteur à s'interroger sur l'œuvre qu'il lit, sur le statut qu'il convient de lui attribuer.

L'aventure transfictionnelle allographe de Boisgobey avec Lecoq s'est arrêtée ici ${ }^{30}$. L'œuvre ayant connu un beau succès, la décision s'explique sans doute moins du point de vue commercial que créatif. En effet, dans ses récits, Boisgobey explore spécialement le type de l'investigateur plus ou moins compétent et propose une « démocratisation du personnel des enquêteurs ${ }^{31} »$. En d'autres mots, il multiplie et varie les figures chargées de résoudre le meurtre: policier et détective, évidemment, mais aussi militaire (Le Crime de l'opéra), noble désœuvré (L'Affaire mystérieuse, La Main coupée), médecin désirant retrouver sa vie de plaisirs (Les Suites d'un duel), étudiant qui se déguise en mohican à la campagne (Le Coup de pouce), artiste qui manque de sérieux, de tact et de jugement (Le Crime de l'Omnibus). Sous sa plume, n'importe qui devient enquêteur, dont maints personnages réticents et aux compétences discutables. Réutiliser l'un d'entre eux considéré comme un modèle d'efficacité cadre donc difficilement avec une telle approche. Ce qui ne signifie pas que Boisgobey abandonne pour autant la transfiction.

\section{Jeux de noms}

13 Nous avons évoqué que, aux côtés des expansions transfictionnelles allographes dans le roman judiciaire de la fin du XIXe on trouve peu de cas de «transfiction autographe ${ }^{32}$ ». Rares sont les auteurs qui ont créé seuls un univers fictionnel reposant sur un personnage structurant; et aucun n'est comparable à Lecoq ou Holmes. Mentionnons toutefois Fifi Vollard, de Constant Guéroult, un «voyou justicier [qui] apparait dans trois romans savoureux et hauts en couleurs qui connaîtront plusieurs rééditions ${ }^{33}$ » à partir de 1876. Aujourd'hui quelque peu oublié, cet ensemble basé sur un célèbre fait divers - l'assassinat de la femme d'un marchand de la rue du Temple en $1838^{34}-$, qu'a aussi repris Eugène Sue dans ses Mystères de Paris (1842-1843), aurait peut-être pu être développé mais le phénomène ne prend vraiment son envol qu'une trentaine d'années plus tard, notamment avec Arsène Lupin de Maurice Leblanc (1905), Rouletabille de Gaston Leroux (1907), Toto Fouinard de Jules Lermina (douze fascicules entre novembre 1908 et janvier 1909). Le succès remporté par Holmes est bien sûr en cause (cela est bien connu dans le cas de Lupin) mais d'autres facteurs sont à considérer. Isabelle Daunais avance que « la transfictionnalité serait toujours plus ou moins la suite d'une étrangeté première (5) $^{35}$, "étrangeté » qui caractérise bien l'enquêteur du roman judiciaire de la fin du XIX ${ }^{e}$ siècle, systématiquement isolé et singularisé dans les récits. Selon l'hypothèse de Daunais, il serait ainsi fort propice à une telle circulation transfictionnelle.

Loin de négliger la voie de la transfiction autographe, comme la majorité de ses confrères, Boisgobey choisit plutôt de déployer une variété de pratiques onomastiques pour détourner et jouer avec l'idée d'un personnage structurant tout un univers fictionnel. Il est vrai qu'il propose un cas discret d'enquêteur récurrent: le policier 
Jottrat, présent dans deux romans parus à quelques mois d'intervalle dans Le Petit Moniteur Universel du Soir (L'Homme sans nom, 10 juin au 14 août 1869, et Disparu!, 31 décembre 1869 au 19 mars 1870). Ces « apparitions successives [...] s'additionnent et sont compatibles ${ }^{36} »$; l'écrivain attribue un univers fictionnel commun à ces intrigues en faisant de ce " "retour [de] personnag[e]" [...] un puissant facteur d'unitée ${ }^{37}$ ». Cependant, Boisgobey explore aussi l'autre extrémité du spectre : la répétition de noms pour des personnages que les récits interdisent de confondre. Le comte Golymine est un voleur qui meurt au début du Crime de l'opéra (1879) - sans qu'il soit possible qu'il ait échappé à la mort - et une victime qui décède dans Le Cri du sang (1885 $\left.5^{38}\right)$. De même, un Roger Pontac décède dans Le Bac (1881-1882) et un autre Roger Pontac dans Le Plongeur $\left(1889^{39}\right)$. Entre ces deux extrêmes, Boisgobey crée aussi d'autres jeux homonymiques dont voici un cas particulièrement ambigu.

Le lecteur de La Vieillesse de M. Lecoq, publié en 1877 et dont l'intrigue est située en 1875, rencontre un personnage nommé "Piédouche", policier compétent au nom peu commun et acteur important dans l'intrigue. Ce nom était déjà apparu sous la plume de Boisgobey dans Le Coup de pouce, publié en 1874 et relatant des événements de 1870, liés à la menace prussienne. Il resurgit également en 1881 dans Le Crime de l'Omnibus, où l'intrigue se déploie en 1880, et dans Le Coup d'œil de M. Piédouche, publié en 1883 et offrant des péripéties ayant lieu en 1882-1883. Examinons un instant ces personnages.

Le Piédouche du Coup de pouce est un gendarme banal au rôle marginal; celui de La Vieillesse de M. Lecoq est un policier honnête et efficace; il pourrait s'agir du premier, plus expérimenté et maintenant installé à Paris, mais le roman ne l'affirme pas. Lorsque ce nom est employé dans Le Crime de l'Omnibus, les choses se compliquent : il s'agit d'un voleur et d'un assassin. Expert dans l'art de se grimer, il se joue de presque tous les enquêteurs impliqués dans l'affaire ; s'il est finalement arrêté, son sort reste nébuleux. Le nom revient dans Le Coup d'œil de M. Piédouche : il est porté par un agent de recherche louche, au passé mystérieux qui, après quelques errances, fait preuve de perspicacité pour résoudre un meurtre. Il semble bien plus âgé que le précédent - même si les intrigues sont rapprochées dans le temps - mais son talent pour le déguisement pourrait expliquer ceci.

Boisgobey construit ainsi une zone grise. Sándor Kálaï avance qu'en raison de la « grande dissemblance entre ses apparitions, ["Piédouche"] ne peut être considéré comme le même personnage ${ }^{40}$ » mais Thierry Chevrier laisse pour sa part la porte ouverte au parcours rocambolesque ${ }^{41}$ d'un même personnage passionné par les mystères, devenu criminel puis cherchant à se racheter dans Le Coup d'œil de M. Piédouche ${ }^{42}$. Aucun d'eux ne meurt et la chronologie n'exclut pas cette hypothèse. Les personnalités sont différentes mais non absolument incompatibles; on pourrait poser que le gendarme de campagne devient un policier à Paris, puis un criminel pour améliorer son sort et cherche enfin le repentir en aidant à résoudre un meurtre, sans réussir à effacer son passé. Ce scénario n'est pas exposé en toutes lettres mais ne serait en aucun cas le plus invraisemblable proposé par Boisgobey.

Notre objectif ne consiste pas à trancher pour affirmer qu'il s'agit ou non d'« une reprise du nom [...] motivée par des éléments insistant sur la continuité ontologique de l'être désigné ${ }^{43}$ ». Retenons plutôt que Boisgobey répète ce nom peu banal, en l'espace de quelques années, pour des personnages dont deux sont au cœur de l'intrigue et dont un apparaît dans le titre ; l'inattention semble peu probable. Et, surtout, soulignons que ce procédé remet en question l'autarcie des romans: publiés dans des journaux 
distincts, sans autres renvois entre eux, ils sont néanmoins liés par ce nom. Il n'y a ni continuité nette ni contradiction catégorique entre les portraits ; plutôt une invitation à s'interroger sur la possibilité d'arrimer ces œuvres. Pour celui qui l'a déjà rencontré chez Boisgobey, le nom « Piédouche ", sans agir comme une clé interprétative menant à une signification claire, incite à une pratique de décodage potentiellement ludique. Boisgobey greffe un questionnement supplémentaire aux enjeux typiques du roman judiciaire (découvrir l'identité du coupable ou la méthode employée pour le crime, ce qui n'est pas toujours aisé avec cet écrivain). Posant que la lecture comprend ici la résolution de telles ambiguïtés disséminées dans les récits, il ajoute à cette activité une dimension herméneutique.

Il serait donc hasardeux de se borner à considérer ces différents jeux homonymiques présents dans les romans de Boisgobey comme des erreurs : le cas Piédouche laisse voir un travail qui crée une réelle indécidabilité quant à l'existence d'un univers fictionnel commun. Ailleurs, cet écrivain offre un autre cas singulier, cette fois en réunissant des récits sur la base de liens fort ténus.

\section{L'univers de l'Opéra}

20 Le Crime de l'Opéra, un des plus célèbres romans de Boisgobey, parait dans Le Figaro du 30 avril au 13 août 1879, puis chez Plon la même année. Il est republié dans «Le Voleur illustré à partir du 12 mai 1882 [et, à nouveau,] en 1889 chez Plon » et enfin en " septembre 1905 dans le $n^{\circ} 1$ de la revue Les Faits-Divers Illustrés ${ }^{44} »$. Ce parcours, qui n'a rien d'inhabituel pour l'époque, doit être rapproché de deux autres titres : Le Pavé de Paris, publié dans Le Voltaire du 28 décembre 1880 au 26 février 1881, puis chez Plon la même année, et Le Mari de la diva, qui paraît dans Le Figaro du 21 mai au 12 juillet 1884, puis chez Dentu la même année et chez Taillandier en 1916 et en $1922^{45}$. En effet, les intrigues de ces trois romans sont liées entre elles et forment un ensemble romanesque qui se matérialise en un volume en 1885 , lorsque les éditions Rouff publient un ouvrage intitulé Le Crime de l'Opéra où les deux premières parties sont formées du Crime de l'Opéra de 1879 et les deux suivantes du Pavé de Paris et du Mari de la diva. Ce récit de 1200 pages, dont nous ignorons le processus de création ${ }^{46}$, constitue de ce fait une mise en œuvre particulière de lecture sérielle, comme on va le voir.

21 Composé de deux parties, Le Crime de l'opéra de 1879 est un roman judiciaire développé autour d'un meurtre où la coupable échappe aux autorités et s'exile après avoir été démasquée par un des héros. Dans Le Pavé de Paris, troisième partie de l'édition Rouff, une femme entretenue, qui jouait un rôle accessoire dans les parties précédentes, est au centre d'une rivalité amoureuse impliquant quatre personnages et est assassinée; ce crime et l'enquête qui s'ensuit organisent le récit. Seule cette " horizontale ", comme on appelait alors les femmes gagnant ainsi leur vie, unit l'intrigue aux parties antérieures, le reste du personnel romanesque est renouvelé. À la fin du récit, les deux rivaux périssent lors d'un duel et ne survit à l'hécatombe que la véritable coupable, laquelle est au cœur de la quatrième partie où elle ruine un homme qui a le même prénom qu'une victime du duel concluant le volet précédent. Dans les derniers chapitres, un fonctionnaire rapporte la conduite de cette femme fatale et les événements de la troisième partie ; son nom et son portrait incitent à reconnaître en lui un juge d'instruction présent dans les deux parties initiales, ce qui complète l'arrimage ténu entre ces intrigues, situées ainsi dans un même univers fictionnel. 
résumé donne à voir diverses similitudes au sein de cet ensemble: rivalités amoureuses mortelles, présence d'une meurtrière, échec des policiers, sauf à la toute fin $\mathrm{du}$ dernier récit, enquêteurs réticents. Les premières parties relèvent du roman judiciaire et la dernière davantage du roman de mœurs. Néanmoins, on note surtout que l'univers fictionnel ainsi créé ne s'appuie pas sur un personnage structurant, comme Rocambole par exemple; il s'agit plutôt d'un «cas limite» de " transfictionnalité ne reposant que sur un détail minime ${ }^{47} »$ : un personnage secondaire des deux premières parties est la victime dans la troisième, sa meurtrière est au cœur de cette partie et de la quatrième, et celui qui rendra possible le châtiment final de la coupable dans cette dernière partie est un personnage rencontré dans la première.

Ajoutons que Boisgobey n'a pas utilisé l'appareil paratextuel ou les publicités dans les journaux concernés pour afficher ou revendiquer cet ensemble comme un projet plus imposant qu'un roman isolé. Pour découvrir l'univers fictionnel composé par ces œuvres, il faut toutes les lire au hasard ou dans l'édition Rouff, puisque rien n'indique préalablement à la lecture qu'elles sont liées. Ceci ne constitue donc pas un argument de séduction en amont; c'est au fil des pages que le lecteur reconnaît ces personnages et réalise que ces récits forment un tout. Ce processus s'apparente à une "annexion transfictionnelle rétrospective ${ }^{48}$ ", un phénomène que Richard Saint-Gelais étudie notamment avec Si par une nuit d'hiver un voyageur d'Italo Calvino. Certaines de ses observations s'appliquent bien à notre ouvrage ; il constate ainsi que l'œuvre :

multiplie les signes de non-continuité entre les romans [...] : indépendance des intrigues, rang secondaire des personnages réapparaissant, caractère généralement allusif des passages qui les mentionnent (de sorte qu'on se demande plus d'une fois s'il s'agit bien des mêmes personnages $)^{49}$.

C'est ce qui est en jeu avec Le Crime de l'opéra, où la façon dont Boisgobey élabore son univers fictionnel opère une sélection au sein des lecteurs, distinguant ceux qui ont lu l'édition Rouff ou les trois romans, et les autres ${ }^{50}$. Cette approche illustre à son tour le caractère particulier de la démarche de Boisgobey, comme l'ont fait la réutilisation du nom Piédouche et les jeux avec Lecoq. S'il procède dans chaque cas d'une manière qui le singularise parmi ses confrères, une cohérence se dégage des stratégies employées.

La capture transfictionnelle de Lecoq, l'arrimage ambigu de romans distincts avec la reprise de noms propres et la transfictionnalité limite visant un groupe exclusif de lecteurs marquent tous une sorte de «franchissement métaleptique ${ }^{51}$ ». Même s'il n'est pas question d'écrivain pénétrant dans la fiction ou de personnage en sortant, la notion de métalepse s'avère ici pertinente: Richard Saint-Gelais rappelle que Françoise Lavocat a montré « la parenté que la transfictionnalité [...] entretient avec les pratiques métaleptiques, paradoxales et réflexives ${ }^{52} »$. Gérard Genette a par ailleurs spécifié que la métalepse ne se résume pas à la "métaphore d'auteur ", qu'elle "s'étend à bien d'autres modes de transgression, figurale ou fictionnelle, du seuil de la représentation ${ }^{53}$ ». Dans le cas qui nous occupe, Boisgobey instaure une fiction qui invite le lecteur familier de son œuvre à dépasser les bornes de chaque livre pour déterminer lui-même l'articulation à établir entre plusieurs d'entre eux.

C'est-à-dire que les procédés déployés ici imposent au lecteur de décider s'il fait ou non un « raccord discursif aux conséquences éminemment déstabilisantes - [ce qui, le cas échéant, contribue] à faire [de lui] un complice de la métalepse ${ }^{54} »$. Ce n'est pas sans conséquence : que la présence de cette dernière résulte « ou non d'un dessein délibéré de la part des écrivains, [elle] ten[d] à instituer une lisibilité nouvelle et autre, à ce titre

Belphégor, 19-1 | 2021 
concurrentielle des modes de lecture par identification ${ }^{55}$ ". L'enjeu consistant à établir ou non ce " raccord discursif » apparaît clairement dans l'ambiguïté de l'usage du nom Piédouche. Dans le cas de la création d'un univers fictionnel unissant trois ouvrages qui semblent isolés (hors de l'édition Rouff), l'ambiguïté n'a pas la même ampleur mais le lecteur, s'il peut ne pas tenir compte du " raccord », ne peut en prendre conscience que durant la lecture, modifiant sa conception du livre alors qu'il est en train de le lire (il doit repenser ce roman comme faisant partie d'un ensemble). Enfin, la relation créée entre les Lecoq de Boisgobey et de Gaboriau pose explicitement le « raccord» mais le rend problématique en en faisant une relation entre une fiction et un texte. Chacun de ces procédés constitue ainsi « un mécanisme par lequel la fiction tente de prendre en charge une opération qui excède son ordre ${ }^{56} »$.

Afin de bien cadrer ces observations, rappelons ici un constat crucial formulé par Richard Saint-Gelais lorsqu'il explique que de tels phénomènes :

ne sont guère commandés par un programme théorique, mais sont bien davantage requis pour réguler un certain nombre de fonctionnements internes de ces fictions provocatrices et déstabilisantes, ne serait-ce que sur un mode ludique ${ }^{57}$.

Ceci décrit avec justesse l'œuvre de Boisgobey où l'on discerne, entre autres éléments " provocateurs", une tendance à brouiller les limites fictionnelles, narratives et textuelles des romans.

En fait, Boisgobey déploie une stratégie qui évoque le fonctionnement de la littérature sérielle où :

non seulement les œuvres s'inscrivent dans un ensemble plus large de textes qui leur donnent sens (ce qui est vrai de toute production textuelle), mais [où] elles supposent d'être appréhendées par la médiation de cette série, puisque c'est à partir de ses conventions que nous sommes invités à déchiffrer l'ouvrage que nous avons entre les mains ${ }^{58}$.

Si Boisgobey ne propose pas une série créée par une collection ou par la récurrence d'un personnage, il crée bel et bien "un pacte de lecture sériel spécifique ${ }^{59}$ ", encourageant la remise en question de l'autarcie de ses romans. En vertu de la diversité des supports, des lieux et des moments de parution, ces stratégies ne sont pas destinées au lectorat d'un journal ou à celui d'une maison d'édition, mais au "lecteur de Boisgobey", celui qui tente de le suivre dans ses publications malgré leur éparpillement. Ainsi, ces jeux constituent autant de procédés structurants qui s'ajoutent à sa signature pour relier et articuler ses œuvres.

31 Reprenons les choses autrement. Plusieurs des cas où un personnage romanesque prend une telle ampleur qu'il semble phagocyter son auteur concernent des écrivains associés au roman judiciaire ou policier : celui de Sherlock Holmes est le plus connu, mais on peut aussi évoquer ceux d'Arsène Lupin, "Rouletabille ou Chéri-Bibi, Fantômas ${ }^{60}$ ». À propos des œuvres qui les donnent à lire, Jean-Claude Vareille affirme que, alors, le « lecteur n'écoute pas un auteur ; il est habité par un Texte, hanté par lui ${ }^{61}$ ». Nous retenons cette remarque car Boisgobey tend à s'extraire de ce paradigme, et ce, même s'il a recours à plusieurs des procédés employés par les créateurs de ces personnages célèbres. Au lieu de structurer clairement plusieurs œuvres entre elles, par exemple au moyen d'un tel personnage (ce qui présente un réel intérêt pour un feuilletoniste), Boisgobey reprend ces procédés en les détournant et les retournant sur eux-mêmes, jusqu'à ce qu'ils en viennent plutôt à évoquer sa propre signature, sa propre manière d'écrire. 

Boisgobey n'a pas ménagé ses efforts pour faire preuve d'inventivité en proposant des crimes commis d'une manière originale et en faisant du dévoilement du coupable un rebondissement inattendu. Cet effort pour surprendre le lecteur apparaît également, on l'a vu, dans le recours à des pratiques transfictionnelles: les cas abordés ici d'expansion et de capture transfictionnelles, de transfictionnalité ambiguë issue de jeux homonymiques et de transfictionnalité limite se rejoignent en ce qu'ils incitent à s'interroger sur la possibilité d'arrimer des romans autrement épars. Si divers contemporains de Boisgobey ont joué avec «ce verrou, cette frontière intangible et intimidante entre les textes, [pour le faire] sauter [avec] la transfictionnalité ${ }^{62} »$, la spécificité de son travail réside dans cette approche qui engage aussi le lecteur à se questionner sur les modalités d'un tel arrimage.

Dans les romans de Boisgobey, où une des principales constantes est la présence d'enquêteurs errant doublement - dans leurs solutions et dans l'espace ${ }^{63}-$, on trouve évidemment divers emprunts à Gaboriau, relevés notamment par Régis Messac et Roger Bonniot. Cependant, le ton léger et les pratiques de Boisgobey ne doivent pas occulter le fait que se développent ici de réelles remises en question et qu'en émerge une conception singulière du roman judiciaire. Celle-ci est marquée par un caractère ludique qui la distingue notamment de l'œuvre de Gaboriau, pour ne citer que l'auteur de romans judiciaires le plus connu de l'époque. Ce ludisme anime ainsi différents procédés déployés dans ces œuvres; retenons d'une part la mise en scène inusitée de certains personnages, et tout particulièrement de l'enquêteur qui fait l'objet d'un traitement distancié, et, d'autre part, les interrogations quant au statut des romans évoqués. La rencontre de ces deux phénomènes contribue à créer une oscillation dans la manière de lire ces œuvres, notamment en ce qui a trait à la possibilité ou à la nécessité de les "prendre au sérieux». En émergent l'effet de "modernité » et le caractère "décalé ${ }^{64}$ » des récits de Boisgobey. Ses romans judiciaires illustrent des possibilités que les noms conservés par l'histoire du genre n'ont pas retenues mais qui ne sont pas pour autant dénuées d'intérêt. Pour surprendre son lecteur, Boisgobey se livre souvent à des expérimentations qui peuvent passer inaperçues si elles sont prises isolément mais qui se révèlent autrement stimulantes lorsqu'elles sont pensées collectivement, les unes avec les autres. Il rappelle ainsi, avec éloquence, qu'un bon roman judiciaire ne doit jamais être lu seul ${ }^{65}$.

\section{NOTES}

1. Sir Arthur Conan Doyle, Ma vie aventureuse, trad. de Louis Labat, Rennes, Terre de Brume, 2003, p. 111. Bruno Monfort a déjà mis en évidence ce passage («Sherlock Holmes ou le plaisir de la “non-histoire” ", Poétique n 101, fév. 1995, p. 49 ; cité par Anne Besson, D’Asimov à Tolkien. Cycles et séries dans la littérature de genre, Paris, CNRS Éditions, «CNRS littérature », 2004, p. 20).

2. L'Affaire Lerouge (1865), Le Crime d'Orcival (1866), Le Dossier 113 (1867), Les Esclaves de Paris (1868) et Monsieur Lecoq (1869), pour la publication en volume. 
3. À ce sujet, voir notamment l'ouvrage d'Elsa de Lavergne intitulé La Naissance du roman policier français. Du Second Empire à la Première Guerre mondiale (Paris, Classiques Garnier, "Études littéraires du Xx ${ }^{\mathrm{e}}$ et $\mathrm{xxI}^{\mathrm{e}}$ siècles », 2009) et celui d'Andrea Goulet intitulé Legacies of the Rue Morgue (Philadelphie, Presses de l'Université de Pennsylvanie, 2016). Dans ce dernier, Goulet expose l'artificialité d'une séparation étanche entre le récit judiciaire du XIX ${ }^{\mathrm{e}}$ siècle et le roman policier classique caractérisé par des « énigmes logiques et des conventions formelles » du siècle suivant (p. 196 ; notre traduction).

4. Notre utilisation de ces appellations suit le travail d'Anne Besson qui explique que «[1]a série insiste davantage sur l'indépendance de ses volumes, qui forment un ensemble discontinu; le cycle, lui, insiste davantage sur la totalité réalisée par l'ensemble, en instaurant une continuité entre ses volumes. Ces principes constituent la traduction, en termes de structure et de forme, d'une ambition différente pour chaque ensemble : la réitération pour la série, la totalisation pour le cycle » (D'Asimov à Tolkien, op. cit, p. 22).

5. Richard Saint-Gelais, Fictions transfuges. La Transfictionnalité et ses enjeux, Paris, Seuil, " Poétique ", 2011, p. 71. Nous emprunterons à cet ouvrage sa typologie des phénomènes transfictionnels.

6. Lise Queffélec, Le Roman-feuilleton français au XIX $X^{e}$ siècle, Paris, Presses universitaires de France, «Que sais-je? », nº 2466, 1989, p. 94.

7. Ibid.

8. Elsa de Lavergne, La Naissance du roman policier français, op. cit., p. 322. Voir aussi Lise Queffélec, Le Roman-feuilleton français au XIX ${ }^{e}$ siècle, op. cit., p. 93.

9. Pour un portrait biographique plus détaillé, voir l'ouvrage déjà cité d'Elsa de Lavergne ( $L a$ Naissance du roman policier français, op. cit., p. 311-316), ainsi que deux textes de Thierry Chevrier : la préface à la réédition du roman Le Coup d'œil de M. Piédouche (Paris, Payot \& Rivages, «Rivages/ Mystères », 1999, surtout p.14-23), et l'article "Histoire d'une redécouverte. Fortuné du Boisgobey, le Gaboriau méconnu " (Le Rocambole. Bulletin des amis du roman populaire, nouvelle série, n 1, printemps 1997, p. 25-29).

10. Thierry Chevrier, « Histoire d'une redécouverte. », art. cit., p. 29.

11. Les informations concernant la publication feuilletonesque des romans de Boisgobey sont tirées du panorama détaillé établi par Thierry Chevrier ("Bibliographie», Le Rocambole. Bulletin des amis du roman populaire, nouvelle série, $\mathrm{n}^{\circ} 1$, printemps 1997, p. 36-49).

12. Sylvain Larue, Les Grandes Affaires criminelles de Paris, Paris, De Borée éditions, 2007, p. 193-201. 13. Maurice Leblanc, Arsène Lupin contre Herlock Sholmès, Paris, Lafitte, 1908. Contrairement à Sholmès, Tolbiac, bien qu'il soit un enquêteur doué, est le véritable maître d'œuvre de la conspiration.

14. Une anecdote rapportée par Thierry Chevrier donne à penser qu'il a parfaitement réussi : lorsque le roman a été publié dans L'Indépendant de Dour en Belgique, les «droits d'auteur furent attribués à Émile Gaboriau, erreur dont on ne devait s'apercevoir que l'année suivante » (" Bibliographie ", art. cit., p. 49).

15. Il se plaît à «bless[er] son chef » en attaquant son orgueil (Émile Gaboriau, L'Affaire Lerouge, Paris, Éditions du Masque, « Labyrinthes », 2004, p. 21).

16. «Cet homme, aux allures de chef de bureau, n'était autre qu'un illustre employé de la préfecture de police, M. Lecoq.» (Émile Gaboriau, Dossier 113, Paris, Éditions du Masque, « Labyrinthes ", 2004, p. 58).

17. Émile Gaboriau, Monsieur Lecoq, Paris, Éditions du Masque, «Labyrinthes », 2003, p. 17.

18. Fortuné du Boisgobey, La Vieillesse de M. Lecoq, Édition Jacques Olliveau, Mi Li Ré Mi (impression à la demande Lulu.com, 2014 [1878], t. I, p. 43.

19. Ibid., t. I, p. 252. Ce surnom, contrairement à ce que le roman laisse entendre, renvoie chez Gaboriau non à Lecoq mais à son mentor, le père Tabaret dans L'Affaire Lerouge (Émile Gaboriau, op. cit., p. 21). 
20. Gévrol (L'Affaire Lerouge, Monsieur Lecoq) ; Pâlot et le tailleur Van Klopen (Le Dossier 113, Les Esclaves de Paris), la marquise d'Arlange et Claire (L'Affaire Lerouge, Monsieur Lecoq), Toto Chupin (Monsieur Lecoq, Les Esclaves de Paris). Voir l'ouvrage de Roger Bonniot Émile Gaboriau ou La Naissance du roman policier (Paris, J. Vrin, 1985, notamment p. 195-196) et l'article «Pratiques sérielles dans le roman judiciaire. Le cas de Gaboriau» de Sándor Kálaï (Belphégor, Littérature populaire et culture médiatique, $\mathrm{n}^{\circ} 14$ (« Sérialités »), 2016. URL : http :/ /journals.openedition.org/ belphegor/696 (consulté le 8 octobre 2019).

21. Cette liste a été établie par Thierry Chevrier («Postérité littéraire de Gaboriau : Résurrections de Monsieur Lecoq", Le Rocambole. Bulletin des amis du roman populaire, vol. 64/65 «Enquête sur Gaboriau ", automne-hiver 2013, p. 163-191).

22. Ibid., p. 180.

23. Thierry Chevrier, «Bibliographie », art. cit., p. 40.

24. Le « Detective Novel » et l'influence de la pensée scientifique, Paris, Honoré Champion, 1929, p. 521 ; cité dans Roger Bonniot, Émile Gaboriau ou La Naissance du roman policier, op. cit., p. 385.

25. Roger Bonniot, Émile Gaboriau ou La Naissance du roman policier, op. cit., p. 385.

26. Richard Saint-Gelais, Fictions transfuges, op. cit., p. 71.

27. Fortuné du Boisgobey, La Vieillesse de M. Lecoq, op. cit., t. I, p. 32.

28. Richard Saint-Gelais, Fictions transfuges, op. cit., p. 234.

29. Ibid.

30. Nous n'avons pu lire tous les romans de Boisgobey mais il serait étonnant qu'il ait récidivé avec $\mathrm{M}$. Lecoq sans chercher à mettre en évidence la présence de ce personnage en l'annonçant dans le titre.

31. Sándor Kálaï, « Hybridité du récit d'enquête chez Fortuné du Boisgobey », Romantisme, 2010/3, $\mathrm{n}^{\circ} 149$, p. 60-61.

32. Daniel Aranda, «Personnage récurrent et transfictionnalité ", dans René Audet et Richard Saint-Gelais (dir.), La Fiction, suites et variations, Montréal et Rennes, Nota Bene et Presses universitaires de Rennes, 2007, p. 254.

33. Elsa de Lavergne, La Naissance du roman policier français, op. cit., p. 322. Il s'agit du « Drame de la rue du Temple et [des] Exploits de Fifi Vollard (1876), réunis par la suite sous le titre de L'Affaire de la rue du Temple (1879), et La Bande à Fifi Vollard (1880) » (ibid.). Guéroult « consacre [deux décennies plus tôt] une série fleuve aux bas-fonds de Paris : Les Vautours de Paris en collaboration avec le marquis de Foudras (1854), Les Étrangleurs de Paris en collaboration avec Paul de Couder (1859), Les Abîmes de Paris (1865) et Les Damnés de Paris (1867)» (ibid.), projet qui témoigne de son intérêt pour les ensembles romanesques.

34. Le 5 juin 1838, lors d'un vol, les forçats libérés Soufflard et Lesage assassinèrent sauvagement la femme Renault, qui tenait avec son mari un commerce de matelas. L'affaire eut un large retentissement à l'époque et le procès fut particulièrement suivi. Soufflard et Lesage faisaient partie d'une bande de malfaiteurs accusés d'une série de vols à Paris, où on retrouvait notamment une très belle femme et un gamin criminel qui ont nourri la création des personnages de Fleur-de-Marie et de Tortillard des Mystères de Paris. À ce sujet, on peut par exemple consulter Armand Fouquier, Causes célèbres de tous les peuples, Paris, H. Lebrun, 1858, $10^{\mathrm{e}}$ livraison (" Soufflard et Lesage »).

35. «Condition du personnage transfictionnel», dans René Audet et Richard Saint-Gelais (dir.), La Fiction, suites et variations, op. cit., p. 355.

36. Anne Besson, D’Asimov à Tolkien, op. cit., p. 71.

37. Ibid., p. 69.

38. Elsa de Lavergne, La Naissance du roman policier français, op. cit., p. 47.

39. Ibid.

40. Sándor Kálaï, « Hybridité du récit d'enquête chez Fortuné du Boisgobey », art. cit., p. 59.

41. Thierry Chevrier, «Préface », art. cit., p. 23-24. 
42. Compte tenu de la relative discrétion de ces occurrences, il est possible que d'autres romans de l'abondante production de Boisgobey comportent aussi « un » Piédouche.

43. Matthieu Letourneux, "Le récit de genre comme matrice transfictionnelle », dans René Audet et Richard Saint-Gelais (dir.), La Fiction, suites et variations, op. cit., p.75.

44. Thierry Chevrier, « Bibliographie », art. cit., p. 40.

45. Ibid., p. 41-42.

46. Il nous a été impossible de déterminer si Boisgobey avait planifié d'entrée de jeu une «trilogie » ou s'il s'agissait plutôt de choix successifs consistant à rattacher les deux romans au Crime de l'opéra et si l'éditeur Rouff a joué un rôle quelconque dans ce processus. Nous ne pouvons donc pas nous avancer davantage sur la création de ce Crime de l'opéra - celui publié par Rouff en 1885 - ; notre réflexion ne concerne que la lecture que l'on a pu faire de cet ensemble une fois qu'il a été constitué.

47. Matthieu Letourneux, «Le récit de genre comme matrice transfictionnelle », art. cit., p. 73.

48. Richard Saint-Gelais, « Contours de la transfictionnalité », dans René Audet et Richard SaintGelais (dir.), La Fiction, suites et variations, op. cit., p. 10.

49. Ibid, p. 11.

50. À la lumière de cette idée d'une récompense réservée aux lecteurs attentifs, on pourrait associer ces indices aux " easter eggs ", fonctions cachées ou clins d'œil discrètement intégrés dans des jeux vidéo ou des films.

51. Jean-Philippe Gravel, Desseins et débris: pour une compréhension pratique des enjeux de la métafiction, mémoire de maîtrise, Université du Québec à Montréal, 2009, p. 14.

52. Selon Richard Saint-Gelais, «Contours de la transfictionnalité », art. cit., p. 21.

53. Gérard Genette, Métalepse, Paris, Seuil, « Poétique », 2004, p. 14.

54. Richard Saint-Gelais, «Le monde des théories possibles: observations sur les théories autochtones de la fiction ", dans Françoise Lavocat, La Théorie littéraire des mondes possibles, Paris, CNRS éditions, 2010, p. 104.

55. Frank Wagner, « Glissements et déphasages. Note sur la métalepse narrative », Poétique, $\mathrm{n}^{\circ} 130$ (avril 2002), p. 249.

56. Richard Saint-Gelais, «Le monde des théories possibles: observations sur les théories autochtones de la fiction », art. cit., p. 113.

57. Ibid., p. 110.

58. Matthieu Letourneux, Fictions à la chaîne. Littératures sérielles et culture médiatique, Paris, Seuil, «Poétique », 2017, p. 496.

59. Ibid.

60. Jean-Claude Vareille, Le Roman populaire français (1789-1914). Idéologies et pratiques. Le Trompette de la Bérésina, Limoges, Presses Universitaires de Limoges / Nuit Blanche, «Littératures en marge », 1994, p. 174.

61. Ibid.

62. Richard Saint-Gelais, « Contours de la transfictionnalité », art. cit., p. 8.

63. Andrea Goulet insiste sur l'importance pour la représentation du détective de l'inscription dans l'espace (Legacies of the Rue Morgue, op. cit., p. 6-9 et p. 251).

64. Respectivement, «Préface ", art. cit., p. 24 et «Boisgobey, Fortuné du », dans Daniel Compère (dir.), Dictionnaire du roman populaire francophone, Paris, Nouveau Monde éditions, 2007, p. 63.

65. Cet article s'inscrit dans un projet de recherche subventionné par le programme «Savoir » du Conseil de recherches en sciences humaines du Canada (2017-2022). 


\section{RÉSUMÉS}

La récurrence du Lecoq d'Émile Gaboriau et du Sherlock Holmes de Sir Arthur Conan Doyle illustre éloquemment l'importance des constructions transfictionnelles dans le roman judiciaire et le récit policier. Si aucun autre exemple véritablement comparable ne s'impose d'emblée durant la seconde moitié du XIX siècle, on constate que quelques auteurs se sont employés à édifier autrement des ensembles romanesques. Parmi eux, Fortuné du Boisgobey (1821-1891) se distingue tout particulièrement. Il publie entre 1875 et 1885 plusieurs récits qui, loin de fonctionner en autarcie, se convoquent mutuellement et mettent en place des univers fictionnels fondés sur des procédés qui invitent le lecteur à s'interroger sur le statut de ce qu'il lit. Ce faisant, Boisgobey propose une conceptualisation singulière et originale du genre judiciaire.

\section{INDEX}

Mots-clés : roman judiciaire, transfictionnalité, séries populaires, Boisgobey, Lecoq, récit policier

\section{AUTEUR}

\section{NICOLAS GAUTHIER}

Nicolas Gauthier est professeur agrégé au département d'études françaises de I'Université de Waterloo (Canada). Examinant notamment la criminalité dans la littérature du XIXe siècle, il s'intéresse tout spécialement aux auteurs et ouvrages aujourd'hui oubliés. II a fait paraître Lire la ville, dire le crime (PULIM, 2017) et a créé le site Le Rez-de-chaussée (http:// rezdechaussee.uwaterloo.ca/), un répertoire en ligne de romans-feuilletons français publiés entre 1836 et 1881 . Depuis juin 2019, il est responsable du site internet de l'association « Littératures Populaires et Culture Médiatique » (https://lpcm.hypotheses.org/) 